\title{
Prevalence and incidence rates of psoriatic arthritis in central Norway: data from the Nord-Trøndelag Health Study (HUNT)
}

\author{
Mari Hoff, ${ }^{1,2}$ Agnete Malm Gulati, ${ }^{1}$ Pål Richard Romundstad, ${ }^{2}$ Arthur Kavanaugh, ${ }^{3}$ \\ Glenn Haugeberg ${ }^{4,5}$
}

Handling editor Tore K Kvien

${ }^{1}$ Department of Rheumatology, St Olavs Hospital, Trondheim, Norway

${ }^{2}$ Department of Public Health and General Practice, Norwegian University of Science and Technology, Trondheim, Norway

${ }^{3}$ Department of Rheumatology, Allergy, Immunology, UC, San Diego, California, USA ${ }^{4}$ Department of Rheumatology, Hospital of Southern Norway Trust, Kristiansand, Norway ${ }^{5}$ Department of Neuroscience, Norwegian University of Science and Technology, Trondheim, Norway

Correspondence to Dr Mari Hoff, Department of Public Health and General Practice, Norwegian University of Science and Technology, Faculty of Medicine, PB 8905, MTFS, 7491 Trondheim, Norway; mari.hoff@ntnu.no

Received 30 April 2013 Revised 23 July 2013 Accepted 27 July 2013 Published Online First 20 August 2013

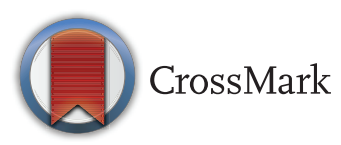

To cite: Hoff M, Gulati AM, Romundstad PR, et al. Ann Rheum Dis 2015;74:60-64.
ABSTRACT

Background $A$ wide range in the prevalence $(<0.01-0.25 \%)$ and incidence (0.5-23.1/100 000) of psoriatic arthritis (PsA) is reported. The main objective of this study was to examine the prevalence and incidence of PsA in central Norway.

Method The patients were recruited from the NordTrøndelag Health Study 3, a population study carried out in 2006-2008. All 94194 inhabitants aged $>20$ years were invited and 50806 (54\%) responded. The study consisted of a questionnaire (Q1) and a brief medical examination. Q1 included questions if the persons suffered from psoriasis, rheumatoid arthritis (RA) or ankylosing spondylitis (AS). Patients with self-reported psoriasis further answered a specific questionnaire on psoriasis including a questionnaire concerning PsA. In order to identify patients with PsA we used the following criteria: Persons reporting they had or may have PsA; persons answering that they had psoriasis and RA; and persons answering that they had psoriasis and AS. Using this approach, 1278 patients were identified. Hospital files were evaluated by a rheumatologist according to a predefined protocol to verify the diagnosis of PsA.

Results 338 patients, 144 men and 194 women, were verified to have PsA. The prevalence of PsA was 6.7 (95\% Cl 5.9 to 7.4 ) per 1000 inhabitants $>20$ years with no significant difference between men and women. In the 9-year period of 2000-2008, a total of 188 patients were diagnosed with PsA, which give an incidence rate of 41.3/100 000 (35.8-47.6).

Conclusions The prevalence of PsA in central Norway appears to be higher than previously reported. The reason for this is unknown and may include environmental factors, life style factors and genetic differences.

\section{BACKGROUND}

Psoriatic arthritis (PsA) is a chronic, inflammatory arthritis associated with psoriasis that may involve peripheral joints, skin and nails, the axial skeleton and entheses. ${ }^{12}$ The clinical presentation of PsA can be heterogeneous. PsA is sometimes classified as a subtype of spondyloarthritis (SpA) based on clinical features, laboratory findings, synovial histopathology, and its association with human leukocyte antigenB27 (HLA-B27) among patients with axial involvement. ${ }^{1} 2$ Extra-articular, extradermatologic manifestations include iridocyclitis and bowel inflammation, similar to those observed in other SpA. ${ }^{2}$
Different classification criteria for PsA have been used over time, which makes it difficult to compare prevalence of PsA across different studies. ${ }^{4} 5$ In 1973, Moll and Wright ${ }^{6}$ defined a set of classification criteria and recognised five different subtypes of clinical presentation in PsA: arthritis in the distal interphalangeal (DIP) joints; asymmetrical monoarthritis or oligoarthritis; polyarthritis; spondylitis; and arthritis mutilans. The classification criteria of Moll and Wright have now been largely replaced by the classification of psoriatic arthritis criteria (CASPAR criteria) from $2006 .^{7}$ In the latter, the presence of inflammation of the entheses is also considered.

Epidemiologic studies have indicated a wide range $(<0.01-0.25 \%)$ in the prevalence of PsA in different countries. ${ }^{8-12}$ The lowest prevalence has been reported in Asia $(<0.01 \%) .{ }^{11} 12$ The highest prevalence has been reported among Caucasians in the USA $(0.25 \%)^{10}$ and in Italy $(0.42 \%) .{ }^{13}{ }^{14}$ In the western part of Norway, Madland and colleagues reported a PsA prevalence of $0.20 \%$ from a hospital cohort. ${ }^{9}$ In a small study among Lapps a prevalence of $0.24 \%$ was found. ${ }^{15}$ The reported incidence ranges from $0.5 / 100000$ in Japan $^{12}$ to 23.1/100 000 in Finland. ${ }^{14} 16$

Genetic differences may provide some explanation of the different prevalences observed for PsA. ${ }^{2}$ Also, as there has not been a standard case definition, different diagnostic methods and classification criteria have been used ${ }^{4}$ Environmental differences may also contribute. Finally, the type of population under study has varied, with some epidemiologic studies reporting prevalences based on hospital cohorts, whereas others report from general population surveys. ${ }^{8}$

The main objective of this study was to examine the prevalence and incidence of PsA in NordTrøndelag. Further, we also examined clinical manifestations and medical treatment of PsA patients.

\section{METHOD}

The patients were recruited from the Nord-Trøndelag Health Study 3 (HUNT 3) performed in 2006-2008. The county of Nord-Trøndelag is located in the central part of Norway and has a population of approximately 128700 , of whom 94194 were above 20 years of age during the study period. The geographic, demographic and occupational structure is fairly representative of the country as a whole. ${ }^{17}$ HUNT has been performed three times. 
In HUNT 3 an invitation letter including the first questionnaire (Q1) was mailed to all adult inhabitants in the county Nord-Trøndelag. Among 94194 invited adults, a total of 50806 (54\%) answered Q1 and participated in the brief medical examination. On the basis of the answers in Q1, people with seven specific disorders received a more detailed questionnaire: these disorders were cardiovascular disease, diabetes mellitus, prostate cancer, colon cancer, breast cancer, eczema and psoriasis. The participants were only allowed to fill out two additional questionnaires and cardiovascular disease was given preference to psoriasis.

In Q1, the following questions about rheumatic diseases were also included, figure 1 :

Do you have rheumatoid arthritis? Yes/No

Do you have ankylosing spondylitis? Yes/No

And, as noted, Do you have psoriasis? Yes/No

2927 patients answered 'yes' regarding psoriasis and further 2006 received the new questionnaire (Q3) about psoriasis. The discrepancy between the two numbers was due to a predefined selection of additional questionnaires such that patients were able to fill out a maximum of two extra questionnaires in addition to Q1, and questionnaires about heart disease and diabetes were given preference to questionnaires about psoriasis.

In Q3 the participants answered the question:

Do you have Psoriasis Arthritis? Yes/No/I do not know.

The respondents in the present study were recruited from HUNT 3 from the following criteria:

1. Persons who answered 'Yes' to the question (Q3 psoriasis): 'Do you have psoriatic arthritis'.

2. Persons who answered 'I do not know' to the question (Q3 psoriasis): 'Do you have psoriatic arthritis'.

3. Persons answering that they had psoriasis and RA (Q1).

4. Persons answering that they had psoriasis and ankylosing spondylitis (AS) (Q1).

5. Due to the predefined selection of only two additional Q3 questionnaires, we also included persons reporting they had psoriasis and cardiovascular disease in Q1, but had not received Q3 and were not included from criteria 3 or 4 . The rationale for this was a theoretical possibility that persons with cardiovascular disease and PsA did not receive the questionnaire about psoriasis as well as they answered 'No' at inclusion criteria 3 and 4 .

This method identified 1238 patients. Over the years, PsA patients in Nord-Trøndelag have either been treated at Namsos Hospital, Levanger Hospital or at St Olavs Hospital. There is no rheumatologist in private practice located in the area.

All medical hospital records from the identified 1238 patient were carefully evaluated by an experienced senior rheumatologist $(\mathrm{MH})$ in order to diagnose PsA according to predefined protocol. To be included, the patents should have been diagnosed of psoriasis verified by a dermatologist or a rheumatologist, as well as arthritis at peripheral joints and/or at spine verified by a rheumatologist. The diagnosis of spinal involvement was based on inflammatory back pain (IBP) and limitation of motion of the lumbar spine. IBP was defined as chronic low back pain that improves with exercise and is not relieved with rest; insidious onset; onset before the age of 40 years and pain at night. ${ }^{4}$ Other rheumatic diseases should be excluded.

In 2000, an electronic medical records system was implemented, thus, the hospital files were evaluated from 2000 up to 2012, however, diagnosis was registered back to 1980 based on medical record information. The evaluation was based on joint involvement, serology, X-ray description, psoriasis in skin and described nail involvement. Treatment and organ manifestations as well as $\mathrm{C}$ reactive protein and erythrocyte sedimentation rate were recorded, and the CASPAR criteria were applied.

\section{Statistics}

Prevalence rates were estimated by dividing the cases with PsA by the whole population participating in HUNT 3. Data were stratified for gender and age groups. CIs were calculated by using binominal distribution.

Crude incidence rates (per 100000 person-years) were calculated as the observed number of cases of PsA in the 9-year
Figure 1 Selection of patients. The number is higher than 1238 , as some patients have answered 'yes' to more than one question.

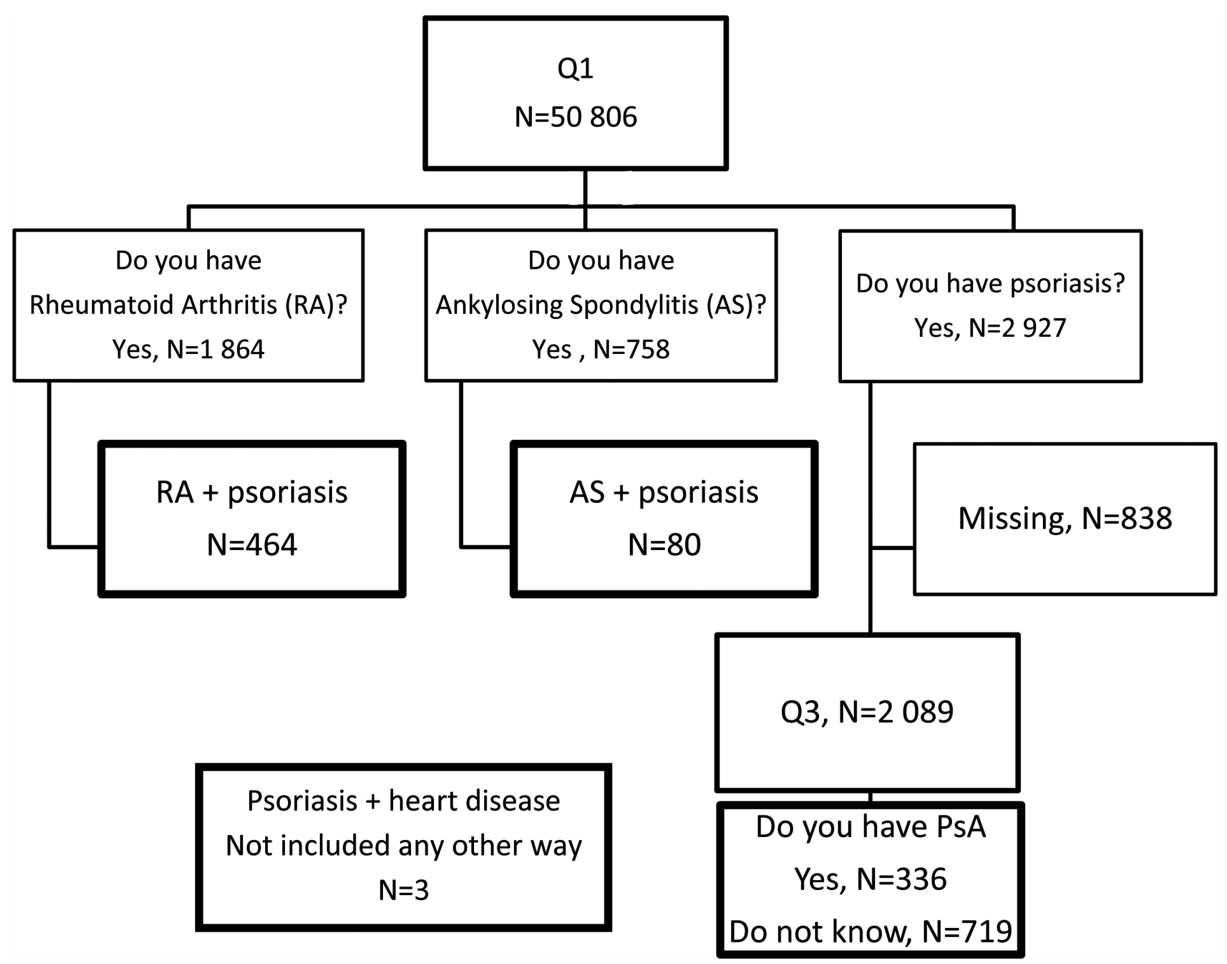


period 2000-2008 divided by the estimated person-time in the same time period. Standardisations were performed by using the 5 -year age distribution of the US 2000 standard population aged 10 years and above (http://seer.cancer.gov).

Descriptive statistics were used to analyse clinical data and treatment. Group analyses were performed with $\chi^{2}$ test.

All tests were two-sided and conducted at the 0.05 significance level.

Statistical analyses were performed using IBM SPSS, V.20 (SPSS, USA) and STATAV.11.0 (StataCorp, College Station, Texas, USA).

\section{RESULTS}

Three hundred and sixty-one patients were verified to have a PsA based on a review of the medical records according to a predefined protocol. Of these, 23 patients were excluded because they were diagnosed after 2008. Table 1 presents the diagnosis among the 1238 patients validated as having PsA.

\section{Prevalence and incidence}

The overall prevalence of PsA was $0.67 \%$ in the Nord-Trøndelag population age above 20 years. Table 2 shows the prevalence stratified for men and women and for different age groups. The highest prevalence was found among women 40-59 years of age.

The positive predictive value for reporting PsA in the questionnaire (Q3) was 68\%.

Totally, 188 patients (80 men and 108 women) were diagnosed with PsA from 2000 to 2008.

The crude incidence rate of PsA in the 9 years from 2000 to 2008 was 41.3/100 000 person-years (95\% CI 35.8 to 47.6). Stratifying for gender the crude incidence rates were 43.4/ 100000 person-years for women (95\% CI 36.0 to 52.5$)$ and 38.7/100,000 person-years for men (95\% CI 31.1 to 48.2).

The age standardised incidence rate (world standard population) of PsA was 35.9/100 000 person-years (95\% CI 30.2 to 41.6).

\section{Classification of psoriatic arthritis criteria (CASPAR criteria)}

Totally, 95.6\% fulfilled the CASPAR criteria while only 15 patients (4.4\%) did not. All these 15 patients had psoriasis and arthritis, but the fact that data on serology were not available most likely contributed to their failure to fulfil the CASPAR

Table 1 Diagnosis among the 1238 patients validated for psoriatic arthritis

\begin{tabular}{ll}
\hline Diagnosis & Number \\
\hline Psoriatic arthritis & 361 \\
& 23 excluded due to diagnosis after 2008 \\
& 338 included in the study \\
Rheumatoid arthritis & 31 \\
Ankylosing spondylitis & 14 \\
Psoriasis and arthralgia & 37 \\
Psoriasis and inflammatory bowel & 15 \\
disease & 30 \\
Psoriasis and fibromyalgia & 8 \\
Gout & 64 \\
Osteoarthritis & 39 \\
Other rheumatic diseases & 639 \\
No rheumatic disease & \\
\hline
\end{tabular}

Table 2 The prevalence $(95 \% \mathrm{Cl}$ ) of psoriatic arthritis (per 1000 inhabitant's age $\geq 20$ years) stratified for gender and age groups

\begin{tabular}{lcc}
\hline Age, years & Men $(95 \% \mathrm{Cl})$ & Women $(95 \% \mathrm{Cl})$ \\
\hline $20-39$ & $3.5(1.8$ to 5.3$)$ & $3.2(1.7$ to 4.6$)$ \\
$40-59$ & $8.1(6.3$ to 9.9$)$ & $10.0(8.2$ to 11.9$)$ \\
$>60$ & $5.5(4.0$ to 7.0$)$ & $5.9(4.4$ to 7.3$)$ \\
Total & $6.7(5.2$ to 7.3$)$ & $7.0(6.0$ to 8.0$)$ \\
\hline
\end{tabular}

criteria. The diagnosis of PsA for all 15 patients was confirmed by two experienced rheumatologists (MH and $\mathrm{GH})$.

\section{Demographic and clinical characteristics}

The demographic and clinical characteristics for the 338 included patients are shown in table 3 , and their use of medications in table 4. Interestingly, 54.9\% had ever used synthetic Disease-modifying antirheumatic drugs (DMARDs) and $17.7 \%$ had ever used anti-tumor necrosis factor alpha (TNF) therapy, the only biologic DMARD approved for PsA in Norway in 2008. There was no difference between ever users of methotrexate (MTX) and sulfasalazine between the genders. More women than men had ever used leflunomide and anti-TNF therapy.

Table 3 Demographic and clinical characteristics for the included 338 psoriatic arthritis patients

\begin{tabular}{|c|c|}
\hline \multicolumn{2}{|l|}{$\mathrm{N}=338$} \\
\hline Female, $\mathrm{n}$ & $194(57.4 \%)$ \\
\hline Age (years) & $54.3(11.9)$ \\
\hline Duration of psoriatic arthritis, (years) & $9.1(7.1)$ \\
\hline Body mass index $\left(\mathrm{kg} / \mathrm{m}^{2}\right)$ & $28.4(4.63)$ \\
\hline Daily smoking & $76(22.5 \%)$ \\
\hline$C$ reactive protein $(g / L)$ & $8.12(9.45)$ \\
\hline Erythrocyte sedimentation rate (ESR) (mm/h) & $12.6(12.9)$ \\
\hline Peripheral joint involvement & $325(96.2 \%)$ \\
\hline Only spinal joint involvement & $13(3.8 \%)$ \\
\hline MRI verified & $1(7.7 \%$ of 13$)$ \\
\hline $\mathrm{CT}$ verified & $5(38.5 \%$ of 13$)$ \\
\hline X-ray & $4(30.7 \%$ of 13$)$ \\
\hline Both peripheral and spinal involvement & $91(26.9 \%)$ \\
\hline Palmoplantaris pustolosis & $27(8 \%)$ \\
\hline Nail involvement & $166(49.1 \%)$ \\
\hline Entesitis & $198(58.6 \%)$ \\
\hline Dactylitis & $73(21.6 \%)$ \\
\hline Colitis & $11(3.3 \%)$ \\
\hline Uveitis & $25(7.4 \%)$ \\
\hline \multicolumn{2}{|l|}{ Serology } \\
\hline RF and/or anti-CCP negative & $238(68.9 \%)$ \\
\hline RF and/or anti-CCP positive & $9(2.7 \%)$ \\
\hline NA & $91(26.9 \%)$ \\
\hline \multicolumn{2}{|l|}{ HLA-B27 } \\
\hline Negative & $41(11.4 \%)$ \\
\hline Positive & $28(7.8 \%)$ \\
\hline NA & $292(80.9 \%)$ \\
\hline Juxta-articular new bone formation on X-ray & $43.2(43.2 \%)$ \\
\hline
\end{tabular}


Table 4 The use of disease modifying antirheumatic drugs and anti-TNF treatment in 338 patients with psoriatic arthritis

\begin{tabular}{lccc}
\hline & Male (n=144) & Female (n=194) & p Value \\
\hline Methotrexate & & & \\
Never (\%) & $71(49.3)$ & $91(46.9)$ & 0.61 \\
Ever (\%) & $69(50.7)$ & $99(51.1)$ & \\
$\quad$ Previous (\%) & $20(13.9)$ & $43(22.2)$ & \\
$\quad$ Current (\%) & $49(34.0)$ & $56(28.9)$ & \\
NA (\%) & $4(2.8)$ & $4(2.1)$ & \\
Leflunomide & & & \\
Never (\%) & $126(87.5)$ & $150(77.3)$ & \\
Ever (\%) & $18(12.5)$ & $46(23.7)$ & \\
$\quad$ Previous (\%) & $10(6.9)$ & $29(14.9)$ & \\
Current (\%) & $8(5.6)$ & $17(8.8)$ & \\
Sulfasalazine & & & \\
Never (\%) & $133(92.4)$ & $169(91.8)$ & \\
Ever (\%) & $9(6.3)$ & $16(8.2)$ & \\
Previous (\%) & $6(4.2)$ & $14(7.2)$ & \\
Current (\%) & $3(2.1)$ & $2(1.0)$ & \\
NA (\%) & $2(1.4)$ & $9(4.6)$ & \\
Anti-TNF treatment & & & \\
Never (\%) & $126(87.5)$ & $149(76.8)$ & \\
Ever (\%) & $18(12.5)$ & $45(23.2)$ & \\
Previous (\%) & $3(2.1)$ & $18(9.3)$ & \\
Current (\%) & $15(10.4)$ & $27(13.9)$ & \\
\hline
\end{tabular}

Statistics are performed between ever users (previous and current) versus never users. ${ }^{*} p<0.05$.

NA, not available.

\section{DISCUSSION}

The main finding in this population-based study from central Norway was a high prevalence of PsA of $0.67 \%$. This is higher than what has previously been reported in two previous studies from Norway $(0.2 \%)^{9} 15$ as well as in reports from other parts of the world $(<0.01-0.42 \%) .{ }^{13} 14$ The gender and age distribution in our study, however, was analogous to the Norwegian study by Madland and colleagues from the western part of Norway. ${ }^{9}$ The prevalence of PsA in our study is more comparable with the prevalence of RA in Norway which, in hospital-based studies, has been reported to be approximately $0.5 \% .^{18}{ }^{19}$ Of note, a more equal prevalence of PsA and RA has also been suggested by some investigators assessing other populations. ${ }^{3} 13$

The majority $(96.2 \%)$ of the PsA patients in this study had involvement of peripheral joints while only $3.8 \%$ had exclusive involvement of the spine. These numbers are in the same range as reported by Madland and colleagues. ${ }^{9}$ However, in the HUNT study, as many as $27.2 \%$ with peripheral involvement in addition had a history of IBP. Other comorbidities associated with SpA were also seen in this study, including 3.3\% of patients with colitis and $7.4 \%$ with uveitis. This emphasises that there can be some relationship between PsA and other diseases considered to be in the SpA group.

Although these data are distinct from previous reports from Norway, one possible explanation for the higher prevalence of PsA in this report may be genetic homogeneity of the population. In a report by Bakland and colleagues, they attributed the high prevalence of AS found in the north of Norway to the high prevalence of HLA-B27, which was found in $16 \%$ of that population. ${ }^{20}$ However, in this study, only $19.2 \%$ of the patients had data available for HLA-B27, and among them, only $7.8 \%$ were HLA-B27 positive. As testing for HLA-B27 would be expected to be obtained most commonly among patients for whom there was a suspicion of $\mathrm{SpA}$, and the prevalence of axial arthritis was $27.2 \%$, this would not likely be an underestimate A study from Iceland has shown an increased risk ratio of 39.2 to first-degree relatives of individuals with PsA; by comparison, using the same techniques in the same Icelandic database, the risk ratio for RA was 4.4. ${ }^{21} 22$ Genome-wide association studies have identified various genes to be associated with PsA, including HLA-B27B and genes encoding IL-13. ${ }^{22}$ The population in HUNT is considered stable, immigration and emigration have been low, supporting a genetic homogeneity of population. ${ }^{17}$

A recently published manuscript has found a marked increase in the prevalence of self-reported psoriasis in the adult cohort in the northern area of Norway, raising from $4.8 \%$ in 1979 to $11.4 \%$ in $2008 .^{23}$ The prevalence of persons reporting a doctor's diagnosis was as high as $9.9 \%$. The authors suggest changes in lifestyle, increasing weight, environmental factors, and an increased awareness of the disease as possible explanations. ${ }^{23}$

Weight gain has also been reported from the first to the last HUNT study. In HUNT 1 (performed 1984-1986) mean body mass index (BMI) was $25.3 \mathrm{~kg} / \mathrm{m}^{2}$ in men and $25.1 \mathrm{~kg} / \mathrm{m}^{2}$ in women, while in HUNT 3 BMI had increased to respectively $27.5 \mathrm{~kg} / \mathrm{m}^{2}$ and $26.9 \mathrm{~kg} / \mathrm{m}^{2}$. Further, in HUNT 3 as many as $75 \%$ of the men and $61 \%$ of the women had BMI above $25.0 \mathrm{~kg} / \mathrm{m}^{2}$ which is the definition for overweight. ${ }^{24}$ In some studies, high weight has been linked to both psoriasis and development of PsA. ${ }^{23} 25-27$

A study from USA by Wilson and colleagues correspondingly reported a rising incidence of PsA over 30 years in both men and women. ${ }^{28}$ They explained that this may be related to a true change in incidence or a greater physician awareness of the PsA diagnosis.

A direct comparison between epidemiological studies of PsA is challenging. Different case definitions for the PsA diagnosis have been used and some cohorts are population based while others are hospital based. In the last few years there has been an increasing interest in understanding the pathogenesis of PsA. Further new medication, for example, TNF inhibitors has improved outcome substantially. ${ }^{29}$ New diagnostic tools, like ultrasound and MRI, have also contributed to an earlier diagnostics. $^{30}$ This has increased the awareness of the PsA disease. ${ }^{3}$

In the present study, 55\% had ever use of synthetic DMARDs and $18 \%$ had ever use of anti-TNF therapy, the only biologic DMARD approved for PsA in Norway in 2008. This indicates that PsA is associated with a high disease burden that necessitates appropriate therapy. By comparison, Madland and colleagues, with their report from 2002, found that 40\% were ever user of synthetic DMARDs and $11 \%$ of anti-TNF therapy. ${ }^{9}$ The increased use of anti-TNF therapy in our study compared with Madland's report may be explained by several factors, including greater awareness of the disease burden among PsA patients with a resultant increase in treatments, a greater acceptance of the use of these agents in PsA patients, and others.

Our data present a high incidence for PsA of 41.2/100 000 . The median lag from the onset of joint pain until diagnosis among these 188 patients was 4-year (25-75 percentile: 1-13 years) To the extent that there may have been greater access to a specialist in rheumatology in 2000-2008 than in previous years, along with greater disease access and more therapeutic options, this may have contributed to the higher incidence observed.

The main limitation for this study was the extent of nonparticipants which may have biased the results. Patients with complaints may have been more likely to participate than 
healthy individuals, thus resulting in a higher prevalence of PsA. However, a study of non-participants in HUNT 3 does not support that there should be a systematic higher prevalence of rheumatic inflammatory diseases among the HUNT participants. ${ }^{31}$ This study of non-participant in HUNT 3 was based on three sources: questionnaire to non-participants; consultations and diagnosis by the general practitioners (GP) in Nord-Trøndelag; and data on dispensed drugs during 2008 from the Norwegian Prescription Database (NorPD) for inhabitants of the Nord-Trøndelag. The prevalence of self-reported RA and AS in HUNT was the same as reported from the GP, unfortunately, PsA was not examined. ${ }^{31}$ Factors that favoured non-participation were older age, the presence of mental disease and lower socioeconomic status. ${ }^{31}$

On the other hand, the prevalence of PsA could, theoretically, be an underestimation among the HUNT participants. Some people suffering from PsA among the participants may have answered they did not have psoriasis. Further, due to a selection where patients only were able to fill out maximum of two extra questionnaires in addition to Q1 as mentioned in the 'Method part', 828 patients with self-reported psoriasis did not answer the question about PsA. However, by using broad inclusion criteria for possible PsA, 146 of these persons were included and 62 were found to have PsA.

Another limitation may be that patients with longstanding disease not using DMARDs, may only have been followed by the primary care. We only had the complete electronic medical records system from 2000, however, diagnosis was registered back to 1980 based on medical record information. Patients were only included if we had sufficient data to verify the diagnosis of PsA, and the prevalence may, therefore, have been underestimated.

Among the identified PsA patients, 96\% of the patients fulfilled the CASPAR criteria; less than $5 \%$ of patients did not fulfil the criteria, however, all these patients had arthritis and psoriasis.

The conclusion of this study is that the prevalence of PsA is high in central Norway, with prevalence comparable with RA. The reason for this is unknown and may include environmental factors, life style factors, genetic differences, as well as more awareness of the disease during the last decades.

Acknowledgements The Nord-Trøndelag Health Study (the HUNT study) is collaboration between the HUNT Research Centre, Faculty of Medicine, the Norwegian University of Science and Technology (NTNU); Norwegian Institute of Public Health; and the Nord-Trøndelag County Council. The Research Department at Levanger Hospital has supported practical help regarding the hospital files.

Contributors $\mathrm{MH}$ was the main investigator for the project and prepared the manuscript. AMG and AK contributed to statistical analysis and interpretation of results. PRR contributed to the method and the statistical analysis. GH contributed to the method, statistical analysis and interpretation of results. All the authors substantially contributed to the manuscript. All authors read and approved the final manuscript.

Funding This work has been supported by an unrestricted research grant from Pfizer. GH has received an unrestricted grant from Pfizer, Institutional Protocol number: \#Ws1948273.

Competing interests GH: Supported by an unrestricted research grant from Pfizer.

Ethics approval The study was approved by the regional committee for ethics and medical research in Norway.

Provenance and peer review Not commissioned; externally peer reviewed.

\section{REFERENCES}

1 Kruithof E, Baeten D, De Rycke L, et al. Synovial histopathology of psoriatic arthritis, both oligo- and polyarticular, resembles spondyloarthropathy more than it does rheumatoid arthritis. Arthritis Res Ther 2005;7:R569-80.
2 Baeten $\mathrm{D}$, Breban $\mathrm{M}$, Lories $\mathrm{R}$, et al. Are spondylarthritides related but distinct conditions or a single disease with a heterogeneous phenotype? Arthritis Rheum 2013;65:12-20.

3 Gladman DD. Psoriatic arthritis. Dermatol Ther 2009;22:40-55.

4 Rudwaleit M, Taylor WJ. Classification criteria for psoriatic arthritis and ankylosing spondylitis/axial spondyloarthritis. Best Pract Res Clin Rheumatol 2010; 24:589-604.

5 Eder L, Gladman DD. Psoriatic arthritis: phenotypic variance and nosology. Curr Rheumatol Rep 2013;15:316.

6 Moll JM, Wright V. Psoriatic arthritis. Semin Arthritis Rheum 1973;3:55-78.

7 Taylor W, Gladman D, Helliwell $\mathrm{P}$, et al. Classification criteria for psoriatic arthritis: development of new criteria from a large international study. Arthritis Rheum 2006;54:2665-73.

8 Setty AR, Choi HK. Psoriatic arthritis epidemiology. Curr Rheumatol Rep 2007:9:449-54

9 Madland TM, Apalset EM, Johannessen AE, et al. Prevalence, disease manifestations, and treatment of psoriatic arthritis in Western Norway. J Rheumatol 2005;32:1918-22.

10 Gelfand JM, Gladman DD, Mease PJ, et al. Epidemiology of psoriatic arthritis in the population of the United States. J Am Acad Dermatol 2005;53:573.

11 Tam LS, Leung YY, Li EK. Psoriatic arthritis in Asia. Rheumatology (Oxford) 2009:48:1473-7.

12 Hukuda S, Minami M, Saito T, et al. Spondyloarthropathies in Japan: nationwide questionnaire survey performed by the Japan Ankylosing Spondylitis Society. J Rheumatol 2001;28:554-9.

13 Salaffi F, De Angelis R, Grassi W. Prevalence of musculoskeletal conditions in an Italian population sample: results of a regional community-based study. I. The MAPPING study. Clin Exp Rheumatol 2005;23:819-28.

14 Alamanos $Y$, Voulgari PV, Drosos AA. Incidence and prevalence of psoriatic arthritis: a systematic review. J Rheumatol 2008;35:1354-8.

15 Falk ES, Vandbakk 0. Prevalence of psoriasis in a Norwegian Lapp population. Acta Derm Venereol Suppl (Stockh) 1993;182:6-9.

16 Savolainen E, Kaipiainen-Seppanen O, Kroger L, et al. Total incidence and distribution of inflammatory joint diseases in a defined population: results from the Kuopio 2000 arthritis survey. J Rheumatol 2003;30:2460-8.

17 Krokstad S, Langhammer A, Hveem K, et al. Cohort profile: the HUNT Study, Norway. Int J Epidemiol. Published Online First: 9 Aug 2012. doi: 10.1093/ije/ dys095

18 Kvien TK, Glennas A, Knudsrod OG, et al. The prevalence and severity of rheumatoid arthritis in Oslo. Results from a county register and a population survey. ScandJRheumatol 1997;26:412-18.

19 Uhlig T, Kvien TK, Glennas A, et al. The incidence and severity of rheumatoid arthritis, results from a county register in Oslo, Norway. JRheumatol 1998:25:1078-84.

20 Bakland G, Nossent HC, Gran JT. Incidence and prevalence of ankylosing spondylitis in Northern Norway. Arthritis Rheum 2005;53:850-5.

21 Karason A, Love TJ, Gudbjornsson B. A strong heritability of psoriatic arthritis over four generations-the Reykjavik Psoriatic Arthritis Study. Rheumatology (Oxford) 2009;48:1424-8

22 Bluett J, Barton A. What have genome-wide studies told us about psoriatic arthritis? Curr Rheumatol Rep 2012;14:364-8.

23 Danielsen $\mathrm{K}$, Olsen $\mathrm{AO}$, Wilsgaard $\mathrm{T}$, et al. Is the prevalence of psoriasis increasing? A 30 year follow-up of a population-based cohort. British J Dermatol 2013;168:1303-10.

24 Krokstad S, Knudtsen MS. Public health development The HUNT Study, Norway HUNT 1 (1984-86) - HUNT 2 (1995-97)—HUNT 3 (2006-08) [Folkehelse i endring: Helseundersøkelsen Nord-Trøndelag : HUNT 1 (1984-86)—HUNT 2 (1995-97)-HUNT 3 (2006-08)]. 2011:213s.: ill.; $30 \mathrm{~cm}$.

25 Soltani-Arabshahi $\mathrm{R}$, Wong $\mathrm{B}$, Feng BJ, et al. Obesity in early adulthood as a risk factor for psoriatic arthritis. Arch Dermatol 2010;146:721-6.

26 Russolillo A, lervolino S, Peluso R, et al. Obesity and psoriatic arthritis: from pathogenesis to clinical outcome and management. Rheumatology (Oxford) 2013:52:62-7.

27 Mease P. Update on treatment of psoriatic arthritis. Bull NYU Hosp Jt Dis 2012;70:167-71

28 Wilson FC, Icen M, Crowson CS, et al. Time trends in epidemiology and characteristics of psoriatic arthritis over 3 decades: a population-based study. J Rheumatol 2009:36:361-7.

29 Mease PJ. Psoriatic arthritis: update on pathophysiology, assessment and management. Ann Rheum Dis 2011;70(Suppl 1):i77-84.

30 Tan AL, McGonagle D. Psoriatic arthritis: correlation between imaging and pathology. Joint Bone Spine 2010;77:206-11.

31 Langhammer A, Krokstad S, Romundstad P, et al. The HUNT study: participation is associated with survival and depends on socioeconomic status, diseases and symptoms. BMC Med Res Methodol 2012;12:143. 